\title{
Diagnosis and treatment trends in mucopolysaccharidosis I: findings from the MPS I Registry
}

\author{
Kristin D'Aco • Lisa Underhill • Lakshmi Rangachari • \\ Pamela Arn • Gerald F. Cox • Roberto Giugliani • \\ Torayuki Okuyama $\cdot$ Frits Wijburg $\cdot$ Paige Kaplan
}

Received: 8 November 2011 / Accepted: 29 November 2011 /Published online: 11 January 2012

(C) The Author(s) 2012. This article is published with open access at Springerlink.com

\begin{abstract}
Our objective was to assess how the diagnosis and treatment of mucopolysaccharidosis I (MPS I) have changed over time. We used data from 891 patients in the MPS I Registry, an international observational database, to analyze ages at symptom onset, diagnosis, treatment initiation, and treatment allocation (hematopoietic stem cell transplantation, enzyme replacement therapy with laronidase, both, or neither) over time for all disease phenotypes (Hurler, Hurler-Scheie, and Scheie syndromes). The interval between diagnosis and treatment has become shorter since laronidase became available in 2003 (gap during 2006-2009: Hurler-0.2 year, Hurler-Scheie - 0.5 year, Scheie-1.4 years). However, the age at diagnosis has not decreased for any MPS I phenotype over time, and the interval between symptom onset and treatment initiation remains substantial for both Hurler-Scheie and Scheie patients (gap during 2006-2009, 2.42 and 6.71 years, respectively). Among transplanted patients, an increasing proportion received hematopoietic stem cells from cord blood (34 out of 64 patients by 2009) and was also treated with
\end{abstract}

K. D’Aco $(\bowtie) \cdot$ P. Kaplan

Section of Metabolic Diseases,

Children's Hospital of Philadelphia,

3501 Civic Center Boulevard,

Philadelphia, PA 19104, USA

e-mail: dacok@email.chop.edu

L. Underhill · L. Rangachari · G. F. Cox

Genzyme, a Sanofi Company,

Cambridge, MA, USA

P. Arn

Division of Genetics, Nemours Children's Clinic, Jacksonville, FL, USA

G. F. Cox

Division of Genetics, Children's Hospital Boston, Department of Pediatrics, Harvard Medical School, Boston, MA, USA laronidase (42 out of 45 patients by 2009). Conclusions: Despite the availability of laronidase since 2003, the diagnosis of MPS I is still substantially delayed for patients with HurlerScheie and Scheie phenotypes, which can lead to a suboptimal treatment outcome. Increased awareness of MPS I signs and symptoms by primary care providers and pediatric subspecialists is crucial to initiate early treatment and to improve the quality of life of MPS I patients.

Keywords Mucopolysaccharidosis I $\cdot$ Hurler $\cdot$ HurlerScheie - Scheie - Laronidase - Enzyme replacement therapy · Hematopoietic stem cell transplant

\section{Introduction}

The Mucopolysaccharidosis I (MPS I) Registry was created in 2003 with the purpose of characterizing the natural history and long-term health and treatment outcomes of this rare, life-

\section{R. Giugliani}

Department of Genetics/UFRGS,

Medical Genetics Service/HCPA and INAGEMP,

Porto Alegre, Brazil

\section{T. Okuyama}

Center for Lysosomal Storage Diseases,

National Center for Child Health and Development, Tokyo 157-8535, Japan

\section{F. Wijburg}

Department of Pediatrics, Division of Metabolic Diseases and Amsterdam Lysosome Center "Sphinx,"

Academic Medical Centre, University of Amsterdam,

Amsterdam, Netherlands 
threatening genetic disorder [25]. This report looks at how diagnosis and disease-specific treatment of MPS I have changed over time by analyzing aggregate data from nearly 900 patients enrolled in the MPS I Registry.

MPS I (McKusick 607014) is an autosomal recessive lysosomal storage disorder that is caused by deficient enzyme activity of $\alpha$-L-iduronidase (IDUA), leading to lysosomal accumulation of glycosaminoglycans (GAGs) in multiple tissues throughout the body. MPS I is a chronic, progressive disease affecting the heart, eyes, bones, joints, respiratory system, facial appearance, viscera, and often the central nervous system. It has historically been classified clinically into three syndromes based on age of onset, rapidity of progression, and presence and degree of cognitive involvement [23]. Hurler syndrome describes patients with the most severe form of MPS I, with signs and symptoms typically appearing in infancy and a median age of death of 6.8 years when untreated [20]. Patients with Scheie syndrome present later in childhood and demonstrate slower symptom progression with preservation of cognition and survival into adulthood [30]. Hurler-Scheie describes an intermediate form with no or mild cognitive impairment and death usually occurring in adolescence or early adulthood when untreated. Patients with Hurler-Scheie and Scheie syndromes also have been referred to as having attenuated MPS I. Collectively, these forms represent different degrees of severity along a disease spectrum without strict clinical, biochemical, or molecular diagnostic criteria in place to differentiate them.

With an overall prevalence of 1:100,000 live births [19, 20, 27] and significant variability in presentation, diagnosis of MPS I in all its forms poses a true challenge [8, 32]. Initial diagnosis is primarily based on physician recognition of signs and symptoms. Deficient IDUA activity and excess urinary GAG excretion are seen in all patients, but do not accurately predict disease severity or form. Although some genotypephenotype correlations have been established [11, 29], most known disease-causing mutations (over 100 to date) are individually unique ("private") and uncharacterized. Treatment of MPS I has also proven challenging as disease-specific treatment options are limited, intensive, and not curative. Hematopoietic stem cell transplantation (HSCT) has been used to treat more than 500 patients with MPS I since $1981[1,18]$ and is typically recommended for patients with Hurler syndrome under 2 years of age with normal cognition (DQ>70), as it can prolong survival, preserve neurocognition, and ameliorate some somatic features [21]. However, due to its significant morbidity and mortality, HSCT is reserved for the most severe form of MPS I [5, 6, 28]. The allogenic stem cell infusion is given following conditioning with chemotherapeutic agents used to suppress the immune response; when successful, the transplant is a one-time procedure, though graft failure may necessitate subsequent transplants. Enzyme replacement therapy (ERT) with laronidase (recombinant human $\alpha$-L- iduronidase; Aldurazyme ${ }^{\circledR}$, BioMarin Pharmaceutical and Genzyme, a Sanofi Company) was approved in 2003 to treat the non-neurologic manifestations of MPS I and is the primary treatment option for patients with Hurler-Scheie and Scheie syndromes. Laronidase is also used to treat Hurler patients who are not candidates for HSCT because of age, health status, access to transplant, or parental choice. Laronidase must be given as a weekly peripheral or central intravenous infusion and is a lifelong therapy.

The timing of treatment initiation, and therefore of diagnosis, is thought to be an important factor for the success of both HSCT and laronidase. Developmental outcomes are better when transplant occurs before 24 months of age [26]. Laronidase may also be more beneficial when started early, as suggested by a case report of a sib pair with HurlerScheie syndrome [15]. Other than laronidase and HSCT, additional management of MPS I is symptom-based and largely supportive, such as surgical interventions (e.g., adenotonsillectomy, hernia repair, ventriculoperitoneal shunt, cardiac valve replacement, carpal tunnel release, spinal decompression); physical, occupational, and speech therapies; respiratory support (e.g., continuous positive pressure ventilation with oxygen supplementation); hearing aids; and medications for pain and gastrointestinal disturbances.

With the intent of improving the long-term health outcomes and quality of life of patients with MPS I worldwide, we report how the chronology of symptom onset, diagnosis, and treatment initiation with HSCT and laronidase have evolved over time among Registry patients. We also analyze treatment trends among patients in the MPS I Registry with regard to the use of HSCT and laronidase by reported phenotypes. Although there are some genotype-phenotype correlations in MPS I [29], genotype information was not included in the analysis since treatment decisions are usually based on clinical manifestations, as newborn screening is not yet available.

\section{Methods}

Variables analyzed and statistical methods

Data entered into the Registry as of March 2010 were analyzed. The 891 MPS I patients came from 179 sites in 33 countries (Table 1). Regionally, $46.6 \%$ of the patients came from Europe and the Middle East, 35.1\% from North America, 14.9\% from Latin America, and 3.4\% from Asia Pacific. MPS I forms (Hurler, Hurler-Scheie, Scheie, and unknown phenotypes) were analyzed by year of treatment initiation and year of diagnosis with respect to the following treatment groups: HSCT, ERT with laronidase, both laronidase and HSCT, or neither treatment. A phenotype designation of "unknown" signifies that the reporting physician either checked 
Table 1 Enrollment in the MPS I Registry by region and country

\begin{tabular}{|c|c|c|}
\hline Region & Country & Number of patients \\
\hline \multirow{19}{*}{$\begin{array}{l}\text { Europe and Middle East } \\
\quad(47 \%, n=415)\end{array}$} & Belgium & 9 \\
\hline & Czech Republic & 11 \\
\hline & Denmark & 5 \\
\hline & France & 63 \\
\hline & Germany & 28 \\
\hline & Hungary & 2 \\
\hline & Ireland & 9 \\
\hline & Italy & 26 \\
\hline & Netherlands & 37 \\
\hline & Norway & 1 \\
\hline & Poland & 20 \\
\hline & Portugal & 5 \\
\hline & Russia & 1 \\
\hline & Saudi Arabia & 2 \\
\hline & Slovakia & 1 \\
\hline & Spain & 25 \\
\hline & Sweden & 4 \\
\hline & Turkey & 3 \\
\hline & UK & 163 \\
\hline \multirow[t]{2}{*}{ North America $(35 \%, n=313)$} & Canada & 54 \\
\hline & USA & 259 \\
\hline \multirow[t]{6}{*}{ Latin America $(15 \%, n=133)$} & Argentina & 17 \\
\hline & Brazil & 82 \\
\hline & Chile & 7 \\
\hline & Colombia & 6 \\
\hline & Mexico & 20 \\
\hline & Venezuela & 1 \\
\hline \multirow[t]{7}{*}{ Asia Pacific $(3 \%, n=30)$} & Australia & 1 \\
\hline & Japan & 8 \\
\hline & Korea & 13 \\
\hline & New Zealand & 1 \\
\hline & Singapore & 1 \\
\hline & Taiwan & 6 \\
\hline & & Total 891 \\
\hline
\end{tabular}

"undetermined" or left the field blank on the Patient Enrollment form. Treatment chronology was determined by analyzing age at symptom onset, diagnosis, and first disease-specific treatment (either HSCT or ERT) in relation to reported phenotype and year of diagnosis. These analyses excluded patients who reported symptom onset after diagnosis, as occurs in siblings of children already carrying an MPS I diagnosis, whose diagnosis and treatment chronology are not representative of the general MPS I population. Three time periods were examined: patients diagnosed before 2003 (prior to laronidase approval), patients diagnosed in 2003-2005, and patients diagnosed in 2006-2009. The latter two time periods are not event-specific but were chosen to allow for sufficient numbers of patients in each group for meaningful comparison. Among Hurler patients who underwent HSCT, age at first HSCT was analyzed by year of first HSCT.

Treatment initiation was defined as the initial laronidase infusion or the initial HSCT, whichever treatment modality was used first. Among transplanted patients, the stem cell source (bone marrow, umbilical cord blood, or peripheral blood) was determined. Among transplanted patients receiving laronidase, the timing of laronidase with respect to transplant was analyzed. Peri-transplant ERT was defined as laronidase given at any time during the interval 6 months prior and 3 months after HSCT. With respect to use of ERT in conjunction with transplantation, the distribution of patients by number and location (country) of treatment centers was also determined. Variables are summarized using descriptive statistics, including mean, median, standard deviation, designated percentiles, and minimum and maximum values. As data are not available for all variables in every patient, the number of observations is always designated.

\section{Results}

\section{Patient demographics}

The demographic profiles of the 891 Registry patients are shown in Table 2. Patients classified as having Hurler syndrome made up more than half of the study population, while patients with the Hurler-Scheie and Scheie forms made up one quarter and one tenth, respectively. Caucasian patients made up $>80 \%$ of the Hurler and Scheie groups, but only $61 \%$ of the Hurler-Scheie group. Approximately $9 \%$ of patients had an unknown form. Males and females were equally distributed in this MPS I population, as expected for an autosomal recessive disorder. Consistent with clinical severity, the median ages of symptom onset, diagnosis, and treatment initiation (HSCT or laronidase) were earliest for Hurler patients ( 0.5 year, 0.8 year, and 1.4 years, respectively), intermediate for Hurler-Scheie patients $(1.9,3.8$, and 8.6 years), and latest for Scheie patients (5.4, 9.4, and 17.1 years). The intervals between median age at onset of symptoms to diagnosis and from diagnosis to treatment initiation were on the order of several months for Hurler patients, a few years for Hurler-Scheie patients, and several years for Scheie patients.

Chronology of symptom onset, MPS I diagnosis, and treatment initiation

Figure 1 shows the median age at symptom onset, diagnosis, and initiation of treatment with either HSCT or laronidase for each disease form by year of diagnosis: $<2003,2003-2005$, and 2006-2009. Several notable trends were observed. The 


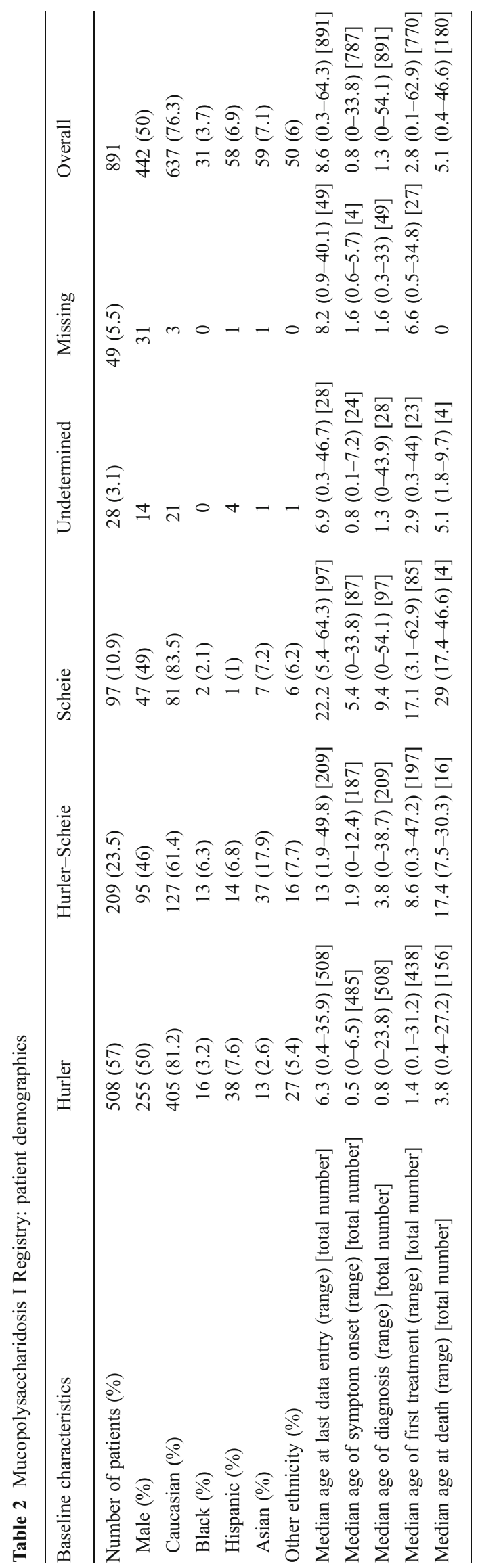

median age at symptom onset remained relatively stable for Hurler and Scheie patients over time, whereas it decreased by approximately 1.5 years for Hurler-Scheie patients after 2003 . On the other hand, the age at diagnosis was stable over time for all forms. The interval between median age at diagnosis and initiation of treatment decreased for all groups after 2003, when laronidase was approved. The decrease was more notable for Hurler-Scheie and Scheie patients (several years) than for Hurler patients (several months). During 2006-2009, the median interval between age at diagnosis and initiation of treatment for individuals with Hurler-Scheie was 0.5 year, and for Scheie patients, 1.4 years. However, even after the 2003 approval of laronidase, the median interval between the age of symptom onset and the age of treatment initiation between 2006 and 2009 for the Hurler-Scheie and Scheie patients was 2.42 and 6.71 years, respectively.

Disease-specific treatment allocation by MPS I phenotype

The allocation of disease-specific treatments over time for the 770 treated patients is shown in Fig. 2. HSCT has been used primarily in Hurler patients, but especially so since 2003. Since laronidase became available, the proportion of patients treated with laronidase has increased in all disease forms. Among Hurler patients, almost half of those who began treatment in 2006-2009 received laronidase alone, and approximately two thirds of those who were transplanted also received laronidase.

\section{Analysis of untreated MPS I patients}

Of the 891 MPS I patients, $116(13 \%)$ were listed as untreated with either laronidase or HSCT. Over time, the proportion of untreated patients has decreased. When analyzed by year of diagnosis, 83 of the 534 patients (16\%) diagnosed before 2003 were untreated, as compared with 21 of the 197 patients (11\%) diagnosed in 2003-2005, and 11 of the 159 patients (7\%) diagnosed in 2006-2009. Among untreated patients, all three disease forms as well as patients with an undetermined/missing phenotype were represented. Of the 115 total untreated patients who had a date of diagnosis, $56(49 \%)$ were Hurler patients diagnosed before 2003 .

HSCT and laronidase treatment trends

Among all patients with Hurler syndrome receiving HSCT, the median age at first transplant has not changed over time (Fig. 3) though the proportion of patients receiving stem cells from cord blood or peripheral blood rather than bone marrow has increased from 26 out of 158 patients $(16.5 \%)$ before 2003 to 33 out of 65 patients in 2003-2005 and 39 out of 64 patients in 2006-2009 (Fig. 4). Among patients receiving stem cells from bone marrow or peripheral blood, the majority of donors were unrelated and the proportion of 
Fig. 1 Median age at symptom onset, diagnosis, and initiation of treatment. Numbers below each bar denote the number of Registry patients in each analysis and year of diagnosis. All data are as of March 2010. Median age is given in years
Hurler

Hurler-Scheie

Scheie

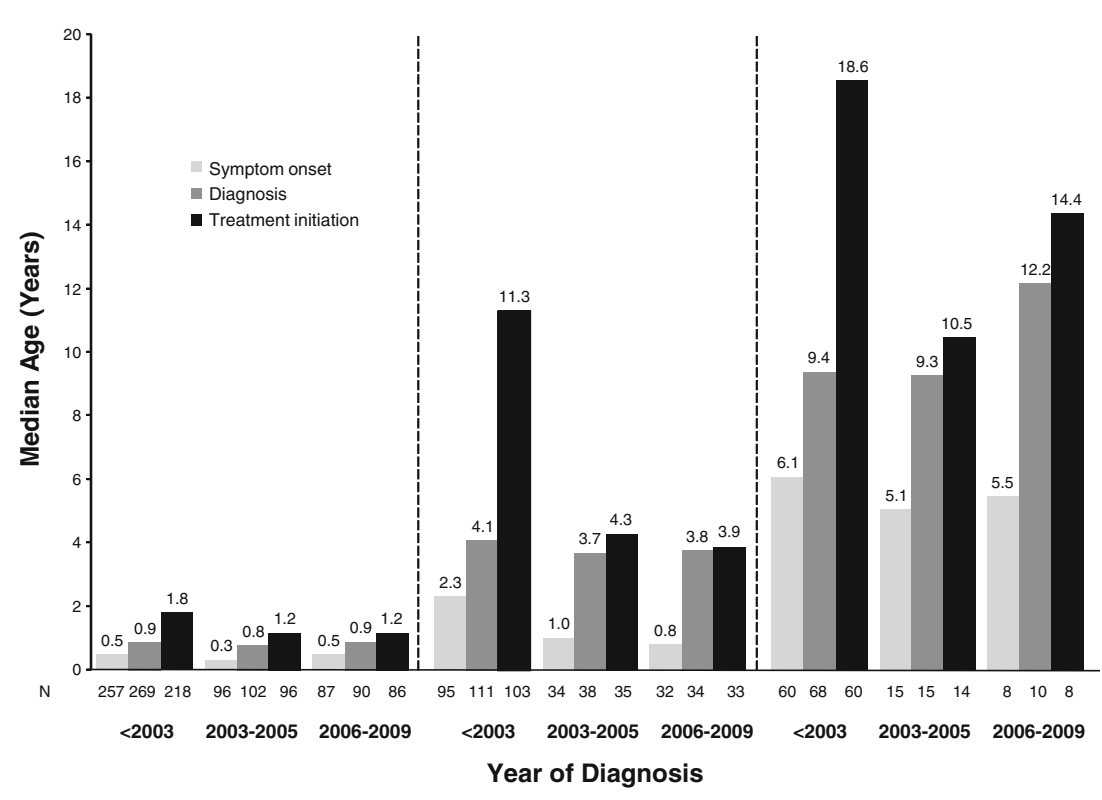

related versus unrelated did not change appreciably over time, although small sample sizes and missing donor information may have masked any trends.
The use of laronidase has greatly increased over time among patients receiving HSCT, the vast majority of whom have Hurler syndrome (Fig. 5). Since 2007, 42 out of 45
Fig. 2 Distribution of treatment modalities over time. Data represent all patients enrolled in the Registry as of March 2010 who report treatment with either HSCT, laronidase, or both. An additional 116 patients reported no treatment
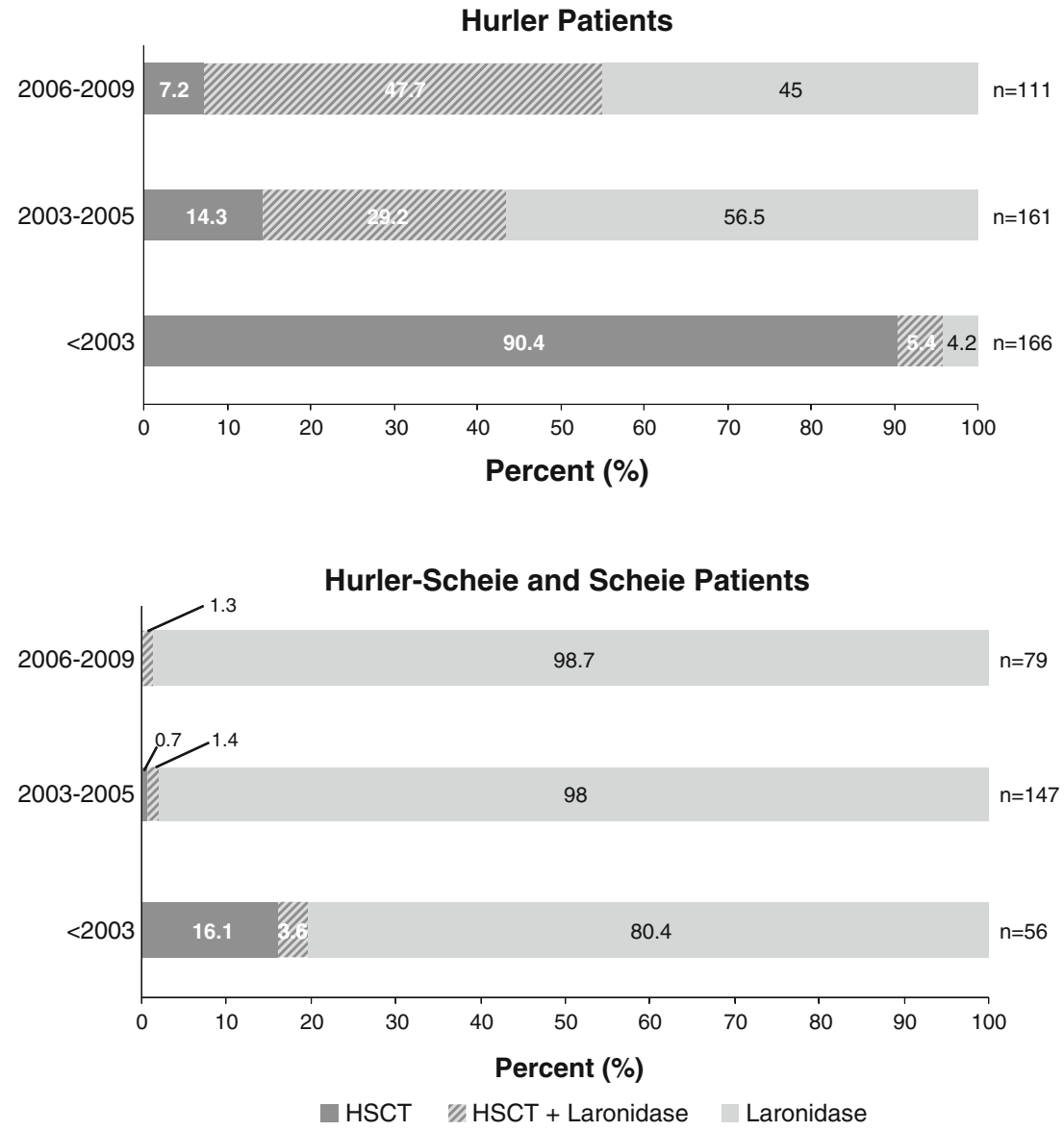


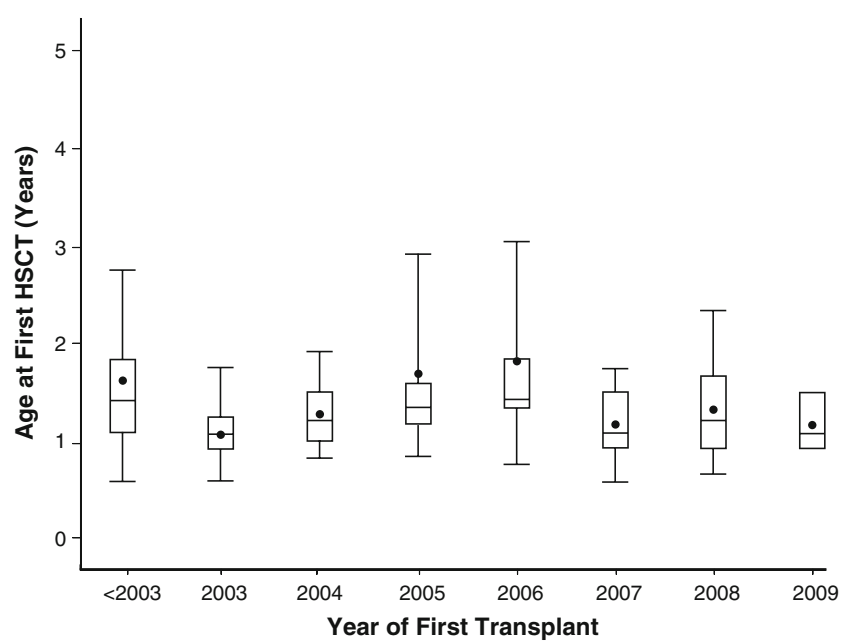

Fig. 3 Median age at first transplant by year of first transplant in Hurler patients. All data are as of March 2010. Horizontal lines within in each box represent the median age, and dots represent the mean age. Lower and upper box edges represent the 25th and 75th percentiles; lower and upper whiskers represent the 5th and 95th percentiles

transplanted patients also reported receiving laronidase. While this analysis included laronidase given to HSCT patients at any time in their disease course, nearly all patients (102 out of $111 ; 91.9 \%$ ) who received both treatments after 2003 received laronidase in the peri-transplant period. The 42 patients who received laronidase and HSCT, with first treatment in 20072009, came from ten centers (18 patients) in the USA and seven centers (24 patients) in Europe (Spain, Belgium, Italy, UK, Netherlands, Czech Republic). Ten of the 17 centers reported only one patient, while the maximum number of patients treated at any one center was 11 . The three patients who underwent their first transplant in 2007-2009 and did not receive laronidase came from different centers in Europe.

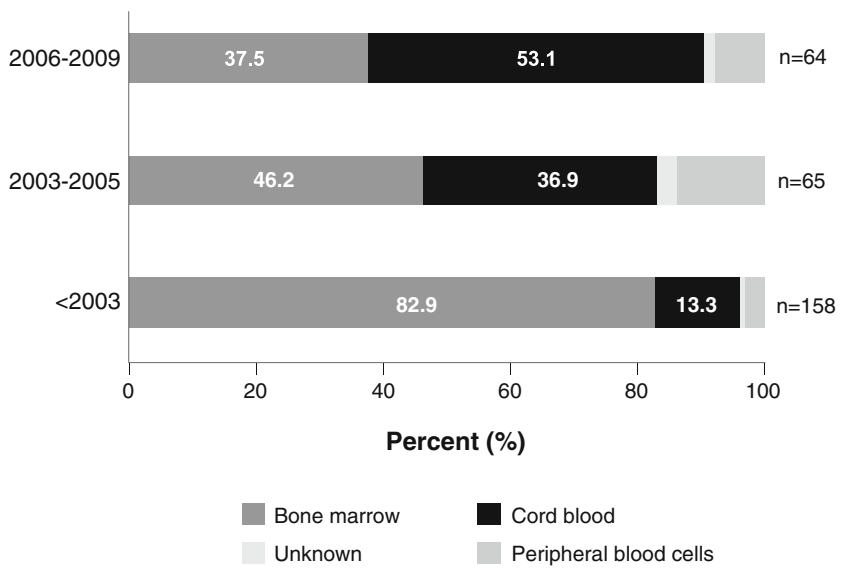

Fig. 4 Hematopoietic stem cell source by year of first HSCT in transplanted patients. Depicted are the relative proportions of various sources of stem cells used for HSCT. All data are as of March 2010

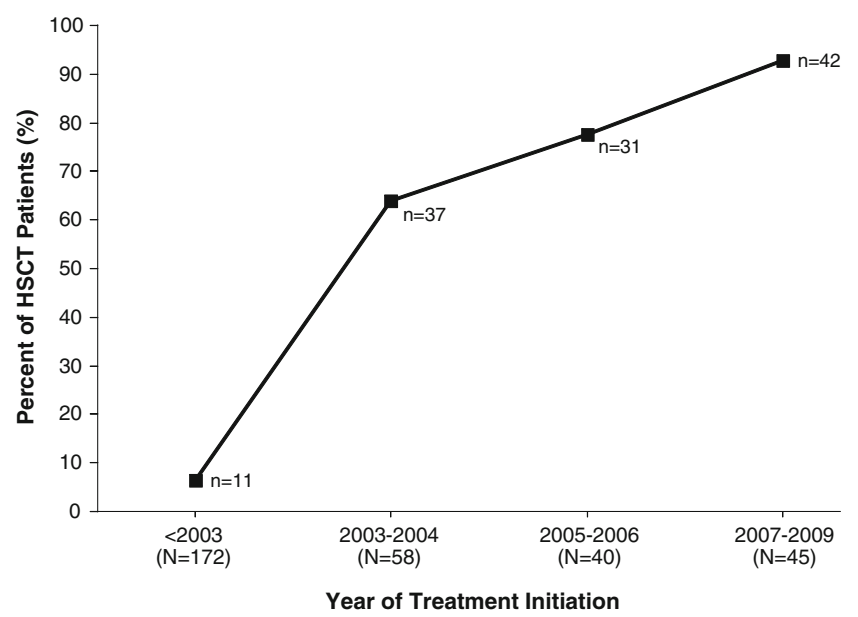

Fig. 5 Use of laronidase with HSCT by year of first treatment. First treatment is defined as either HSCT or laronidase, whichever occurred first. For all treatment periods, $92 \%$ of Registry patients who received both HSCT and laronidase received laronidase during the peritransplant period, defined as any time during the interval 6 months before and 3 months after HSCT. All data are as of March 2010

\section{Discussion}

Analysis of data from the MPS I Registry has allowed for improved understanding of the natural history of MPS I and will enable evaluation of the impact of therapeutic advances on morbidity and mortality $[2,3,25,30]$. Limitations of observational registries such as the MPS I Registry, in which anonymized data are submitted voluntarily, include incomplete, missing, or inaccurate data, as well as losses to follow-up and lack of standardization of patient assessments. The voluntary nature of the Registry may also lead to biased data as not all physicians who manage MPS I patients use the MPS I Registry. Despite these potential biases, the data presented here offer insight into diagnosis and treatment trends in the largest cohort of MPS I patients ever evaluated.

Our data show that the median age at diagnosis has not decreased over time for any form of MPS I, despite available treatment options. Although Hurler patients tend to be diagnosed within a few months of symptom onset, HurlerScheie and Scheie patients still remain undiagnosed for years after symptom onset (Fig. 1). This gap not only represents years of reduced quality of life that would likely be ameliorated by treatment, but also a lost opportunity for preventing or delaying irreversible disease manifestations, as well as for genetic counseling about the risk of future siblings being affected. Sibling case studies and studies in the MPS I dog model suggest that starting enzyme replacement therapy with laronidase shortly after birth can significantly improve clinical outcome [13-15, 33]. Most patients who begin treatment at a very young age have an older sibling with a known diagnosis of MPS I. While studies of affected sib pairs will certainly provide more information 
about the potential impact of early laronidase treatment, prompt clinical diagnosis will be imperative for the majority of MPS I patients to benefit from this information. Demonstration of substantial improvement in clinical outcomes with earlier diagnosis and treatment of MPS I along with an enhanced ability to predict phenotype at birth through genotype-phenotype correlations and/or disease biomarkers will underscore the need for broad implementation of a newborn screening program for this rare, life-threatening disorder and will aid in decisions of which patients to treat with what therapies, and how early to treat them.

As would be expected, the time interval from diagnosis to initiation of disease-specific treatment decreased following the availability of laronidase in 2003, especially for Hurler-Scheie and Scheie patients, who until then had generally been offered only palliative, symptom-based treatments. Prior to 2003, ERT with laronidase was available only through clinical trials, the largest of which enrolled mostly older children, adolescents, and young adults with a mean duration of 13 years since symptom onset [9]. In addition, the proportion of untreated patients has decreased over time. Despite the decrease in time from diagnosis to treatment, there still exists a delay of 0.5 year and 1.4 years between median ages at diagnosis and treatment for Hurler-Scheie and Scheie patients, respectively, during the most recent time interval of 2006-2009. In comparison, for patients with the Hurler form, there was almost no delay between diagnosis and treatment. This may be due to parents and/or diagnosing physicians perceiving the attenuated phenotypes as milder, or less urgent, thereby further delaying time to treatment. In addition, it may represent time needed for laronidase approval by reimbursement authorities [7]. There may also be regional differences in the delay between diagnosis and treatment, given that laronidase was not approved outside the European Union and the USA until 2005.

Interestingly, when looking at Fig. 1, the age of symptom onset appears to have decreased somewhat in both the HurlerScheie and Scheie populations. Rather than a true change in the natural history of MPS I disease, this finding is more likely to be secondary to small patient numbers and the retrospective awareness that certain common early symptoms (such as hernia and chronic otitis media) are often related to MPS I. Also, while the actual age of diagnosis has not greatly improved, parents and physicians may have improved in the retrospective recognition of the common early manifestations of the disease, which led to younger ages of symptom onset in the attenuated patient populations.

Of note, patients with an unknown disease form tended to present with MPS I symptoms between the ages of patients classified as Hurler and Hurler-Scheie. Similarly, they were typically diagnosed and treated at ages in between those of Hurler and Hurler-Scheie patients as well. This suggests that patients in the Registry with an unknown disease form are more likely to be on the more severe end of the MPS I spectrum.
This analysis identified several shifts in clinical practice, such as the increasing use of laronidase among transplanted patients, particularly in the peri-transplant period, and the increasing use of cord blood as a stem cell source. The proportion of unrelated versus related donors for HSCT has remained relatively stable as unrelated bone marrow donors have been replaced by unrelated cord blood donors. Use of laronidase in the peri-transplant period has been reported to be safe and well tolerated without interfering with engraftment or increasing the risk of graft-versus-host disease [6, 12, 16, 17, 31, 34]. Laronidase treatment may be particularly beneficial in patients in poor clinical condition prior to HSCT, to help improve their eligibility for transplant and tolerance of the full-intensity transplant conditioning [12, 16, 17, 24, 31].

In addition, an increasing proportion of Hurler patients in the Registry is receiving laronidase alone (Fig. 2), reflecting the fact that HSCT is not available in many parts of the world, while the majority of transplanted patients are from North America and Europe. A recent MPS I Registry analysis comparing patients from Latin America to those from the rest of the world found that less than $1 \%$ of patients in Latin America had been transplanted, compared to $27 \%$ of patients from the rest of the world [22]. Our MPS I Registry patients came from over 30 countries in Europe and the Middle East, North America, Latin America, and Japan and Asia Pacific (Table 1). Regional differences in treatment availability would also impact the ages at treatment initiation as well as the proportion of untreated patients. For example, in Brazil, which has a universal-access public health care system, neither laronidase nor HSCT is covered by government or specialized pharmaceutical programs [7].

Narrowing the gap between symptom onset and MPS I diagnosis is of the utmost importance and largely relies on increased recognition of clinical red flags by community physicians and pediatric specialists managing the many MPS I-related symptoms, such as otolaryngologists, orthopedists, rheumatologists, and ophthalmologists. While there is significant variability of first presenting symptom both within and between phenotypes, certain symptoms have been consistently noted to appear earlier than others in the MPS I population. Coarse facial appearance, abdominal distension, and corneal clouding are among the most common presenting symptoms in children with Hurler syndrome [4, 10]. In the patients with attenuated forms, joint stiffness/contractures, recurrent ENT symptoms, corneal clouding, and umbilical hernias are the most prevalent initial symptoms [4, 30, 32]. In addition, certain constellations of symptoms should raise a physician's suspicion of MPS I. In a 2009 study of surgical procedures in 544 MPS I Registry patients representing all clinical forms, $72 \%$ had had at least one surgical procedure, with a median of 3 to 4 surgeries per patient, and with nearly half of the patients reporting two or more surgeries by age 4 years [2]. Surgeries often appeared unrelated, such as combinations of ear tubes, 
hernia repair, and tendon releases in the same patient, and often preceded the patient's MPS I diagnosis, particularly in the attenuated phenotypes. Many procedures were also performed at ages atypical for the general pediatric population, such as younger ages of tonsillectomy/adenoidectomy, and older ages of hernia repair in patients with MPS I.

Given the rarity of MPS I, increased efforts to educate community pediatricians and pediatric surgical subspecialists on clinical red flags that should prompt a genetics referral is of the utmost importance. An MPS I Registry analysis of early presenting symptoms for each MPS I form is underway to aid the front-line clinicians with earlier symptom recognition. Furthermore, future analysis of MPS I long-term therapeutic outcomes is merited, as large-scale evidence of improved outcomes with earlier diagnosis and treatment would reinforce the need for increased recognition of clinical red flags, allow better understanding of the therapeutic potential of current treatment modalities, and underscore the need for newborn screening for this disorder.

Acknowledgments The authors thank Jessica Kong, Gregory Fagan, and Ying Zhang of the MPS I Registry for expert biostatistical support and Sarah Kulke, MD (Genzyme Global Medical Affairs) for critical review of the manuscript.

Conflicts of interest $\mathrm{KD}$ receives grant funding from Genzyme for research in lysosomal storage diseases; LU, LR, and GC are employees of Genzyme, a Sanofi company, which distributes laronidase (Aldurazyme), a treatment for MPS I; PA receives honoraria and travel expenses to attend scientific meetings from Genzyme; RG receives research grants, travel expenses to attend scientific meetings, and speaker honoraria from Genzyme; TO receives honoraria and travel expenses to attend scientific meetings from Genzyme; FW receives research grants and reimbursement of expenses and honoraria for lectures on lysosomal storage diseases from Genzyme; PK receives honoraria for presentations and board meetings, travel expenses to meetings, and paid and unpaid consultancy work for Genzyme and has been a principal investigator in Genzymesponsored clinical trials.

Open Access This article is distributed under the terms of the Creative Commons Attribution Noncommercial License which permits any noncommercial use, distribution, and reproduction in any medium, provided the original author(s) and source are credited.

\section{References}

1. Aldenhoven M, Boelens JJ, de Koning TJ (2008) The clinical outcome of Hurler syndrome after stem cell transplantation. Biol Blood Marrow Transplant 14:485-498

2. Arn P, Wraith J, Underhill L (2009) Characterization of surgical procedures in patients with mucopolysaccharidosis type I: findings from the MPS I Registry. J Pediatr 154(859-864):e853

3. Arn P, Whitley CB, Wraith JE, Underhill L, Rangachari L, Cox GF (2011) Postoperative mortality in patients with mucopolysaccharidosis I: findings from the MPS I Registry. J Pediatr Surg (in press)

4. Bodamer OA (2007) Clinical characteristics of MPS I patients in the MPS I Registry. The American Society of Human Genetics, San Diego
5. Boelens JJ, Wynn RF, O'Meara A, Veys P, Bertrand Y, Souillet G, Wraith JE, Fischer A, Cavazzana-Calvo M, Sykora KW, Sedlacek P, Rovelli A, Uiterwaal CS, Wulffraat N (2007) Outcomes of hematopoietic stem cell transplantation for Hurler's syndrome in Europe: a risk factor analysis for graft failure. Bone Marrow Transplant 40:225-233

6. Boelens JJ, Rocha V, Aldenhoven M, Wynn R, O'Meara A, Michel G, Ionescu I, Parikh S, Prasad VK, Szabolcs P, Escolar M, Gluckman E, Cavazzana-Calvo M, Kurtzberg J (2009) Risk factor analysis of outcomes after unrelated cord blood transplantation in patients with hurler syndrome. Biol Blood Marrow Transplant 15:618625

7. Boy R, Schwartz IV, Krug BC, Santana-da-Silva LC, Steiner CE, Acosta AX, Ribeiro EM, Galera MF, Leivas PG, Braz M (2011) Ethical issues related to the access to orphan drugs in Brazil: the case of mucopolysaccharidosis type I. J Med Ethics 37:233-239

8. Cimaz R, Vijay S, Haase C, Coppa GV, Bruni S, Wraith E, Guffon N (2006) Attenuated type I mucopolysaccharidosis in the differential diagnosis of juvenile idiopathic arthritis: a series of 13 patients with Scheie syndrome. Clin Exp Rheumatol 24:196-202

9. Clarke L, Wraith JE, Beck M, Kolodny EH, Pastores G, Muenzer J, Rapoport DM, Berger KI, Sidman M, Cox GF (2009) Long-term efficacy and safety of laronidase in the treatment of mucopolysaccharidosis I. Pediatrics 123:229-240

10. Colville GA, Bax MA (1996) Early presentation in the mucopolysaccharide disorders. Child Care Health Dev 22:31-36

11. Cox GF, Wraith JE, Whitley CB, Wijburg FA, Guffon N (2009) Genotype frequencies in the MPS I Registry. Mol Genet Metab 96: S19 (abstract 31)

12. Cox-Brinkman J, Boelens JJ, Wraith JE, O'Meara A, Veys P, Wijburg FA, Wulffraat N, Wynn RF (2006) Haematopoietic cell transplantation (HCT) in combination with enzyme replacement therapy (ERT) in patients with Hurler syndrome. Bone Marrow Transplant 38:17-21

13. Dickson PI, Hanson S, McEntee MF, Vite CH, Vogler CA, Mlikotic A, Chen AH, Ponder KP, Haskins ME, Tippin BL, Le SQ, Passage MB, Guerra C, Dierenfeld A, Jens J, Snella E, Kan SH, Ellinwood NM (2010) Early versus late treatment of spinal cord compression with long-term intrathecal enzyme replacement therapy in canine mucopolysaccharidosis type I. Mol Genet Metab 101:115-122

14. Dierenfeld AD, McEntee MF, Vogler CA, Vite $\mathrm{CH}$, Chen $\mathrm{AH}$, Passage M, Le S, Shah S, Jens JK, Snella EM, Kline KL, Parkes JD, Ware WA, Moran LE, Fales-Williams AJ, Wengert JA, Whitley RD, Betts DM, Boal AM, Riedesel EA, Gross W, Ellinwood NM, Dickson PI (2010) Replacing the enzyme alpha-L-iduronidase at birth ameliorates symptoms in the brain and periphery of dogs with mucopolysaccharidosis type I. Sci Transl Med 2:60ra89

15. Gabrielli O, Clarke LA, Bruni S, Coppa GV (2010) Enzymereplacement therapy in a 5-month-old boy with attenuated presymptomatic MPS I: 5-year follow-up. Pediatrics 125:e183-e187

16. Grewal SS, Wynn R, Abdenur JE, Burton BK, Gharib M, Haase C, Hayashi RJ, Shenoy S, Sillence D, Tiller GE, Dudek ME, van Royen-Kerkhof A, Wraith JE, Woodard P, Young GA, Wulffraat N, Whitley CB, Peters C (2005) Safety and efficacy of enzyme replacement therapy in combination with hematopoietic stem cell transplantation in Hurler syndrome. Genet Med 7:143-146

17. Hirth A, Berg A, Greve G (2007) Successful treatment of severe heart failure in an infant with Hurler syndrome. J Inherit Metab Dis 30:820

18. Hobbs JR, Hugh-Jones K, Barrett AJ, Byrom N, Chambers D, Henry K, James DC, Lucas CF, Rogers TR, Benson PF, Tansley LR, Patrick AD, Mossman J, Young EP (1981) Reversal of clinical features of Hurler's disease and biochemical improvement after treatment by bone-marrow transplantation. Lancet 2:709-712

19. Meikle PJ, Hopwood JJ, Clague AE, Carey WF (1999) Prevalence of lysosomal storage disorders. JAMA 281:249-254 
20. Moore D, Connock MJ, Wraith E, Lavery C (2008) The prevalence of and survival in mucopolysaccharidosis I: Hurler, Hurler-Scheie and Scheie syndromes in the UK. Orphanet J Rare Dis 3:24

21. Muenzer J, Wraith JE, Clarke LA (2009) Mucopolysaccharidosis I: management and treatment guidelines. Pediatrics 123:19-29

22. Muñoz-Rojas M, Bay L, Sanchez L, van Kuijck M, Ospina S, Cabello J, Martins A (2011) Clinical manifestations and treatment of mucopolysaccharidosis type I patients in Latin America as compared with the rest of the world J Inher Metab Dis 34:10291037

23. Neufeld EF, Muenzer J (2001) The mucopolysaccharidoses. In: Scriver C, Beaudet A, Sly W, Valle D, Childs B, Kinzler K, Vogelstein B (eds) The metabolic and molecular bases of inherited disease. McGraw Hill, New York, pp 3421-3452

24. Orchard PJ, Milla C, Braunlin E, Defor T, Bjoraker K, Blazar BR, Peters C, Wagner J, Tolar J (2009) Pre-transplant risk factors affecting outcome in Hurler syndrome. Bone Marrow Transplant 45:1239-1246

25. Pastores G, Arn P, Beck M, Clarke J, Guffon N, Kaplan P, Muenzer J, Norato D, Shapiro E, Thomas J, Viskochil D, Wraith J (2007) The MPS I registry: design, methodology, and early findings of a global disease registry for monitoring patients with mucopolysaccharidosis type I. Mol Genet Metab 91:37-47

26. Peters C, Shapiro EG, Anderson J, Henslee-Downey PJ, Klemperer MR, Cowan MJ, Saunders EF, deAlarcon PA, Twist C, Nachman JB, Hale GA, Harris RE, Rozans MK, Kurtzberg J, Grayson GH, Williams TE, Lenarsky C, Wagner JE, Krivit W (1998) Hurler syndrome: II. Outcome of HLA-genotypically identical sibling and HLA-haploidentical related donor bone marrow transplantation in 54 children. The Storage Disease Collaborative Study Group. Blood 91:2601-2608

27. Poorthuis BJ, Wevers RA, Kleijer WJ, Groener JE, de Jong JG, van Weely S, Niezen-Koning KE, van Diggelen OP (1999) The frequency of lysosomal storage diseases in the Netherlands. Hum Genet 105:151-156

28. Prasad VK, Kurtzberg J (2010) Transplant outcomes in mucopolysaccharidoses. Semin Hematol 47:59-69

29. Terlato NJ, Cox GF (2003) Can mucopolysaccharidosis type I disease severity be predicted based on a patient's genotype? A comprehensive review of the literature. Genet Med 5:286-294

30. Thomas JA, Beck M, Clarke JT, Cox GF (2010) Childhood onset of Scheie syndrome, the attenuated form of mucopolysaccharidosis I. J Inherit Metab Dis 33:421-427

31. Tolar J, Grewal SS, Bjoraker KJ, Whitley CB, Shapiro EG, Charnas L, Orchard PJ (2008) Combination of enzyme replacement and hematopoietic stem cell transplantation as therapy for Hurler syndrome. Bone Marrow Transplant 41:531-535

32. Vijay S, Wraith JE (2005) Clinical presentation and follow-up of patients with the attenuated phenotype of mucopolysaccharidosis type I. Acta Paediatr 94:872-877

33. Wang RY, Cambray-Forker EJ, Ohanian K, Karlin DS, Covault KK, Schwartz PH, Abdenur JE (2009) Treatment reduces or stabilizes brain imaging abnormalities in patients with MPS I and II. Mol Genet Metab 98:406-411

34. Wynn R, Mercer J, Page J, Carr T, Jones S, Wraith J (2009) Use of enzyme replacement therapy (laronidase) before hematopoietic stem cell transplantation for mucopolysaccharidosis I: experience in 18 patients. J Pediatr 154:135-139 